\title{
Investigating the earliest epochs of the Milky Way halo
}

\author{
Else Starkenburg ${ }^{1}$ and the Pristine Team $\dagger$ \\ ${ }^{1}$ Leibniz Institute for Astrophysics (AIP) \\ An der Sternwarte 16, 18842 Potsdam, Germany \\ email: estarkenburg@aip.de
}

\begin{abstract}
Resolved stellar spectroscopy can obtain knowledge about chemical enrichment processes back to the earliest times, when the oldest stars were formed. In this contribution I will review the early (chemical) evolution of the Milky Way halo from an observational perspective. In particular, I will discuss our understanding of the origin of the peculiar abundance patterns in various subclasses of extremely metal-poor stars, taking into account new data from our abundance and radial velocity monitoring programs, and their implications for our understanding of the formation and early evolution of both the Milky Way halo and the satellite dwarf galaxies therein. I conclude by presenting the "Pristine" survey, a program on the Canada-France-Hawaii Telescope to study this intriguing epoch much more efficiently.
\end{abstract}

Keywords. stars: abundances, Galaxy: evolution, galaxies: abundances, early universe

\section{Introduction}

One field of study that has been very confined to the Milky Way galaxy and some surrounding galaxies is that of individual metal-poor stars. Stars keep a chemical imprint from their birth cloud during their lives and also preserve kinematical information of their infall orbits (if they were born outside of the potential they live in today) for long times. Therefore, studying the present-day stars can teach us about the past and provides a unique opportunity to study galaxy formation in general. Lessons learned from local well-studied stars and stellar systems can be applied to our understanding of the physics of star formation and the assembly of galaxies in the Universe.

The first generations of stars are particularly interesting. As we believe the metallicity of the galaxy is built up over generations, the lowest metallicity stars that still exist today probably represent our closest approach to studying the very first stars. These stars carry the imprint of very few supernovae and it is possible that they have been formed even before the epoch of reionization. At the present day a very limited number of stars are studied - particularly in high-resolution - with an iron-to-hydrogen ratio of less than a thousandth of that of the Sun, i.e., $[\mathrm{Fe} / \mathrm{H}]<-3$ or "extremely metal-poor" (see for some recent overviews and results Aoki et al. 2013; Cohen et al. 2013; Spite et al. 2013; Yong et al. 2013a; Placco et al. 2014). Going even lower, the numbers get more and more limited. At the present day, we know just eight stars with $[\mathrm{Fe} / \mathrm{H}]<-4.5$ (Christlieb et al. 2002; Frebel et al. 2005; Norris et al. 2007; Caffau et al. 2011; Hansen et al. 2014; Keller et al. 2014; Bonifacio et al. 2015), of which four show $[\mathrm{Fe} / \mathrm{H}]<-5$ (hyper

$\dagger$ Thanks to the full Pristine Team: PIs Else Starkenburg \& Nicolas Martin, Co-Is: Piercarlo Bonifacio, Elisabetta Caffau, Raymond Carlberg, Patrick Côté, Patrick François, Stephen Gwyn, Vanessa Hill, Rodrigo Ibata, Julio Navarro, Alan McConnachie, Ruben Sanchez-Janssen \& Kim Venn. Additional thanks to Matthew Shetrone, Alan McConnachie and Kim Venn for their role in the CEMP velocity monitoring survey. 


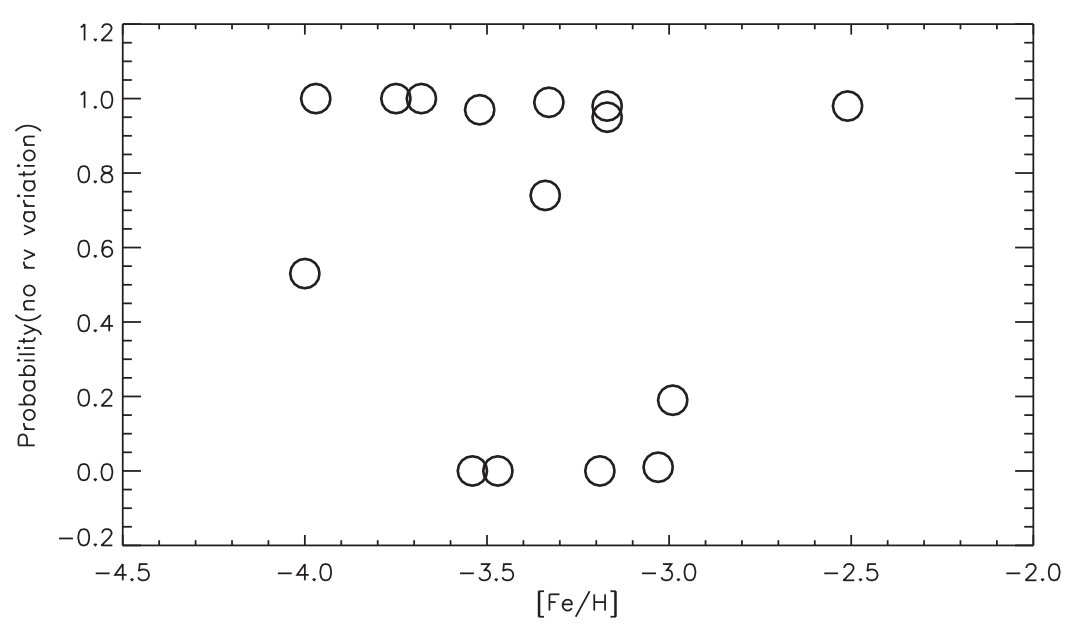

Figure 1. Data taken from Starkenburg et al. (2014, see also references therein) Table 1. For their sample of studied CEMP-no stars, here we plot the probability that the radial velocity observations can be explained by the uncertainty in the radial velocity determination solely. For this purpose, $3 \sigma$ error bars are used (see for a discussion Section 3.2 of Starkenburg et al. 2014). It is clear that although some stars exhibit radial velocity variations, for a majority of the sample the data does not require a binary companion to explain the observations.

metal-poor). These likely belong to the oldest objects we know in the Universe. That we know so few of these very pristine stars that provide such important clues on the earliest stages of star formation is explained by the fact that they are generally vastly outnumbered by stars of later generations in any component of the Galaxy.

\section{Carbon-enhanced metal-poor (CEMP) stars}

Reviewing the sample of extremely metal-poor stars in the Milky Way, it immediately stands out that about a third of these stars show a puzzling high enhancement in carbon (e.g., Yong et al. 2013b). As these stars span a range in stellar parameters and populate both the giant branch and main-sequence (turn-off) branch, it is clear that this carbonenhancement is not produced in the stars themselves. Do these chemical anomalies tell us something important about the very first stages of star formation?

A substantial fraction of CEMP stars also show an overabundance in heavy s-process elements (measured as an enhancement in barium) and are called CEMP-s (Beers \& Christlieb 2005). Stars with low or normal barium values are on the other hand called CEMP-no. A generally accepted explanation across the community is that the CEMP-s stars obtain their overabundant carbon from a companion star that was initially more massive and previously reached the stage of carbon fusion in its interior, dredged this up and has deposited it through winds - along with s-process elements - onto the surface of the extremely metal-poor star. Strong evidence in favour of this scenario was found from repeated radial velocity measurements. The fraction of confirmed binaries in the CEMP-s class is so high that all such stars might indeed be in binary systems (Lucatello et al. 2005, and references therein); thus, the nucleosynthetic signatures of these stars are likely attributable to their binarity.

From a theoretical standpoint, it is insufficiently understood if carbon could be transferred from a companion without s-process elements (Suda et al. 2004), making also CEMP-no stars potentially AGB polluted. To address these issues we have undertaken to monitor the velocities of all CEMP-no stars below $[\mathrm{Fe} / \mathrm{H}]=-3$ from the compilation 
in Norris et al. (2013) in order to understand whether they - or a subsample of them owe their peculiar chemical composition as the result of being in a binary system. From the first analysis of this (partial) dataset observed with the Hobby-Eberly Telescope and presented in Starkenburg et al. (2014) we can already firmly conclude that although there are some clear binaries in this population, most of them show no signature of variations. This is illustrated here in Figure 1. A more statistical analysis, including literature data, shows that the two different classes of CEMP stars - those with high barium and those without - have different binary properties. Through comparison with simulations, we demonstrate in Starkenburg et al. (2014) that all barium-enhanced populations are best represented by a $\sim 100 \%$ binary fraction with a shorter period distribution of at maximum $\sim 20,000$ days. This result greatly strengthens the hypothesis that a similar binary mass transfer origin is responsible for their chemical patterns. The complete CEMP-no dataset is however clearly inconsistent with the binary properties of the CEMP-s class, thereby strongly indicating a different physical origin of their carbon enhancements.

\section{CEMP stars: what do they tell us?}

For the origin of the CEMP-no class an alternative explanation for their chemical pattern - if they indeed are not in binary systems - is that these stars are truly the oldest stars we see and formed from gas clouds already imprinted with a large overabundance of carbon and other light elements by the first stars (see for an overview e.g., Norris et al. 2013; Karlsson, Bromm \& Bland-Hawthorn 2013). Various models predict that gas could be enriched in carbon-rich material by the supernova and/or wind ejecta of fast rotating Pop III stars (e.g., Fryer, Woosley \& Heger 2001; Meynet, Ekström \& Maeder 2006; Cescutti et al. 2013), or supernovae explosions with fall-back, thereby expelling mainly lighter elements (e.g., Umeda \& Nomoto 2003; Limongi, Chieffi \& Bonifacio 2003). The fact that almost all of the most metal-poor stars known are of the CEMP-no class (note however the exception at $[\mathrm{Fe} / \mathrm{H}]=-4.7$ from Caffau et al. (2011)) seems to favor such an explanation. On the other hand, it has been suggested that the most iron-poor stars were in fact born with $[\mathrm{Fe} / \mathrm{H}] \sim-2$, more in line with their carbon abundance pattern, and that their iron abundance has been dust-depleted since (Venn \& Lambert 2008). A follow-up study shows that many of the ultra metal-poor stars do not show any infrared excess, making this interpretation less likely. While for some stars such an interpretation can be ruled out based on their precise abundance patterns, this is not yet the case for all of them (Venn et al. 2014).

A large majority of the extremely metal-poor stars studied today live in a volume fairly close to the Sun (see Figure 2). There are however some indications for a dependence on environment of the number of carbon-enhanced metal-poor stars (and of their type) with either disk height (Frebel et al. 2006); in the inner versus outer halo (Carollo et al. 2014); and in the Galactic halo versus classical dwarf galaxies (Starkenburg et al. 2013; Skúladóttir et al. 2015). However, statistics are lacking and a larger sample of these stars is needed to understand the chemical signatures of the first epoch of star formation and the - perhaps very exotic - phenomena that are proposed to have existed at this early epoch.

\section{Finding the needle in the haystack}

A high fraction of the known extremely metal-poor stars were discovered in the HK and Hamburg/ESO (HES) surveys (Beers, Preston \& Shectman 1985; Christlieb et al. 2002). Both these surveys were centered around the very strong Ca H\&K features in 


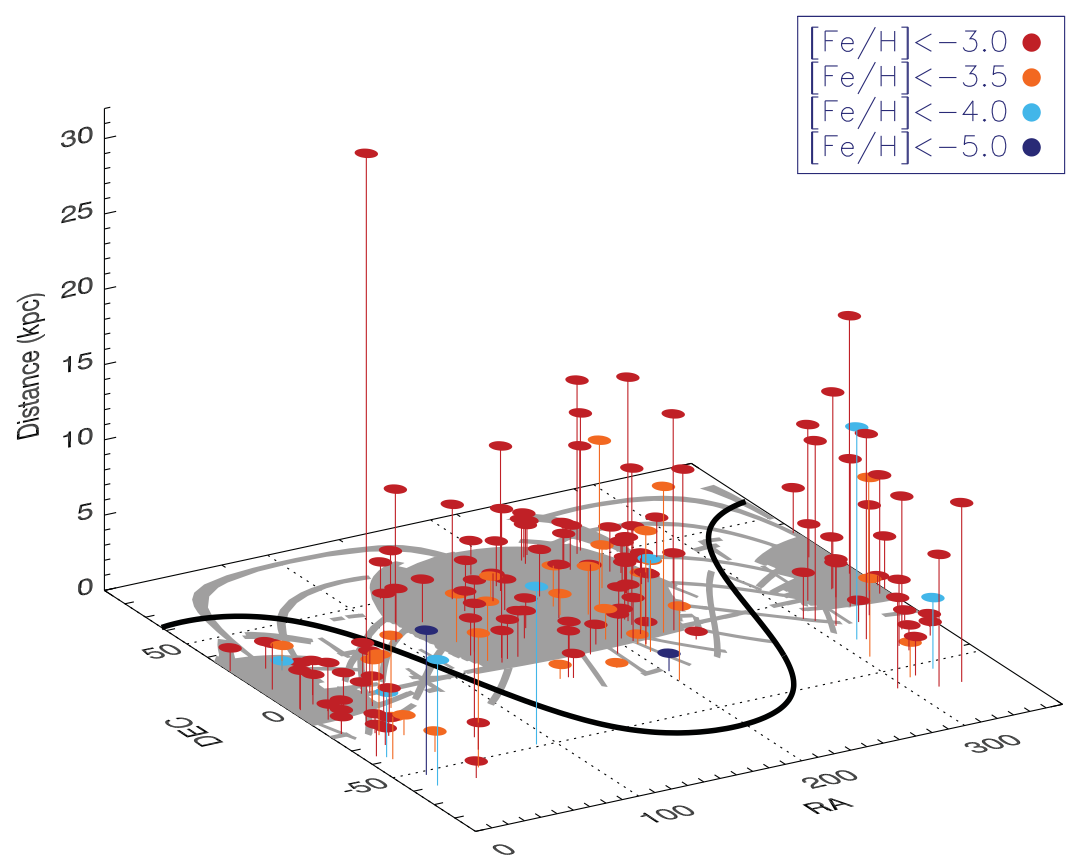

Figure 2. A figure showing the distance from the Sun versus the RA and DEC coordinates for a large sample of extremely metal-poor stars. Distances are taken from the work of Carollo et al. (2014) who have analyzed both the sample from Aoki et al. (2013) and the dataset presented in Yong et al. (2013a). This figure is meant to illustrate how relatively local our knowledge of metal-poor stars still is. The footprint of the Sloan Digital Sky Survey (SDSS) is shown (grey contours) as well as the location of the Milky Way thin disk (black solid line).

the spectrum, using an objective grism technique. Any very metal-poor stars will show hardly any loss of flux in the regions of the broad $\mathrm{Ca} \mathrm{H}$ and $\mathrm{K}$ features (i.e. the lines will be much weaker, the effect is illustrated in Figure 3) setting them apart from more metalrich stars of the same colour. The target lists from these surveys have been dominating the field of research into pristine stars for many years (e.g., see for a recent compilation of results from the HES survey targets Cohen et al. 2013).

Furthermore, the Sloan Digital Sky Survey (SDSS) has significantly contributed to the number of metal-poor stars known in its spectroscopic follow-up mainly carried out as part of the Sloan Extension for Galactic Understanding and Exploration (SEGUE, Yanny et al. 2009). Yet the number of stars with spectroscopic follow-up is a very small fraction of the total area surveyed and its selection function is complex, focussing on many different types of stars. So far $\sim 70$ extremely metal-poor stars from SDSS/SEGUE have been followed with HDS on Subaru (Aoki et al. 2013), a comparable number is currently being followed up with X-shooter on the VLT (Caffau et al. 2013).

\section{CFHT-Pristine}

Utilizing the new narrow-band Ca H\&K filter for MegaCam on the 4-meter CanadaFrance-Hawaii telescope on Mauna Kea the Pristine survey aims to mine a significant area in the footprint of the SDSS in the Northern Hemisphere. The filter is centered around these same $\mathrm{Ca} H \& \mathrm{~K}$ lines illustrated in Figure 3 to allow a very efficient search for metal-poor star candidates. In the first semester the filter has been available, already over $\sim 600$ square degrees of area have been photometrically surveyed. The survey area 


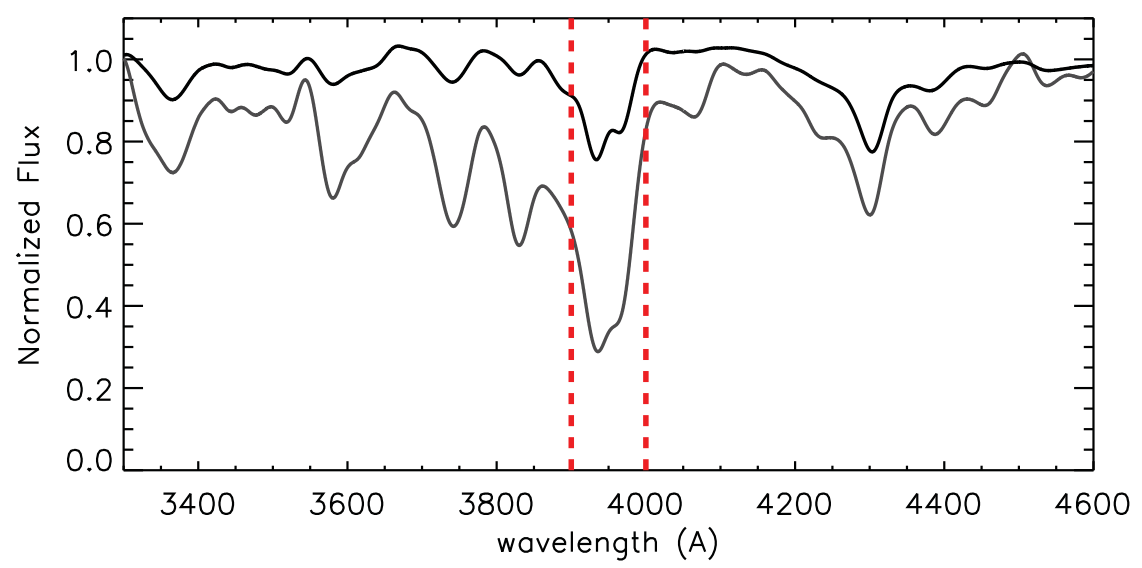

Figure 3. A synthetic spectrum of two red giants with $[\mathrm{Fe} / \mathrm{H}]=-1$ (grey) and $[\mathrm{Fe} / \mathrm{H}]=-3$ (black), made using MARCS models and the Turbospectrum code Alvarez \& Plez (1998). Dashed vertical lines indicate the approximate placing of the boundaries for the Ca H\&K filter. Even in these spectra that are convolved to lower resolution $(\mathrm{R} \sim 1500)$ the $\mathrm{Ca} H \& \mathrm{~K}$ lines clearly stand out as the strongest features in this part of the spectrum and are very sensitive to the stars metallicity. By comparing the flux in such a narrow band filter to that in broader band filters (sensitive to the overall color of the star), stars of different metallicities can be split up easily.

deliberately includes in its footprint a range of galactic latitudes $(30<\mathrm{b}<73)$ and some exciting Galactic substructure features. It includes for instance part of the Sagittarius stellar stream (without being overwhelmed by it). From the first analysis of Pristine photometry, combined with SDSS broad-band colours and results from the overlapping SEGUE spectroscopy (Lee et al. 2008), we can confirm that indeed this new survey is very well equipped to distinguish the sparse extremely metal-poor targets from the more metal-rich bulk of stars in the Milky Way.

We note that this strategy (using a narrow-band Ca H\&K filter in conjunction with broad-band colors) is very similar to the pending SkyMapper survey. SkyMapper is run on an automated wide field 1.35 meter survey telescope at Siding Spring Observatory (e.g., Keller et al., 2007) and is designed to map all of the $20.000 \mathrm{deg}^{2}$ of the Southern Hemisphere in the Sloan-like ugriz filters and additionally a (broader version of a) Ca H\&K filter to find extremely metal- poor stars. From their preparatory investigations and first results overlapping with medium-resolution spectra the SkyMapper Team also finds this filter presents a very clean distinction between the sought for metal-poor stars and the more metal-rich contaminants (Keller, Skymapper Team \& Aegis Team 2012). The Pristine program will be less comprehensive than SkyMapper as we have broad-band colours already available from SDSS. In terms of coverage, the two surveys are very much complementary, as they are directed towards the two different hemispheres.

These narrow-band efforts are expected to alleviate the current lack of statistical samples of extremely metal-poor stars. They will thereby contribute significantly to a new era in Galactic archaeology studies, in particular when combined with other future datasets from the Gaia satellite and upcoming large multi-object spectroscopic surveys.

\section{Bibliography}

\section{References}

Alvarez R. \& Plez B., 1998, A\&A, 330, 1109

Aoki W. et al., 2013, AJ, 145, 13 
Beers T. C. \& Christlieb N., 2005, ARA\&A, 43, 531

Beers T. C., Preston G. W., \& Shectman S. A., 1985, AJ, 90, 2089

Bonifacio, P. et al., 2015, A\&A, 579, A28

Caffau, E. et al., 2011, Nature, 477, 67

-, 2013, A\&A, 560, A71

Carollo D., Freeman K., Beers T., Placco V., Tumlinson J., \& Martell S., 2014, ApJ, submitted, (arXiv:1401.0574)

Cescutti G., Chiappini C., Hirschi R., Meynet G., \& Frischknecht U., 2013, A\&A, 553, A51

Christlieb, N. et al., 2002, Nature, 419, 904

Cohen J. G., Christlieb N., Thompson I., McWilliam A., Shectman S., Reimers D., Wisotzki L., \& Kirby E., 2013, ApJ, 778, 56

Frebel, A. et al., 2005, Nature, 434, 871

-, 2006, ApJ, 652, 1585

Fryer C. L., Woosley S. E., \& Heger A., 2001, ApJ, 550, 372

Hansen, T. et al., 2014, ApJ, 787, 162

Karlsson, T., Bromm, V., \& Bland-Hawthorn, J., 2013, Reviews of Modern Physics, 85, 809

Keller, S. C. et al., 2014, Nature, 506, 463

Keller S. C., Skymapper Team, \& Aegis Team, 2012, in Astronomical Society of the Pacific Conference Series, Vol. 458, Galactic Archaeology: Near-Field Cosmology and the Formation of the Milky Way, Aoki W., Ishigaki M., Suda T., Tsujimoto T., Arimoto N., eds., p. 409

Lee, Y. S. et al., 2008, AJ, 136, 2022

Limongi, M., Chieffi, A., \& Bonifacio, P., 2003, ApJ, 594, L123

Lucatello, S., Tsangarides, S., Beers, T. C., Carretta, E., Gratton, R. G., \& Ryan S. G., 2005, ApJ, 625, 825

Meynet G., Ekström S., \& Maeder A., 2006, A\&A, 447, 623

Norris, J. E., Christlieb, N., Korn, A. J., Eriksson, K., Bessell, M. S., Beers, T. C., Wisotzki, L., \& Reimers, D., 2007, ApJ, 670, 774

Norris, J. E. et al., 2013, ApJ, 762, 28

Placco, V. M., Frebel, A., Beers, T. C., Christlieb, N., Lee, Y. S., Kennedy, C. R., Rossi, S., \& Santucci, R. M., 2014, ApJ, 781, 40

Skúladóttir, Á., Tolstoy, E., Salvadori, S., Hill, V., Pettini, M., Shetrone, M. D., \& Starkenburg, E., 2015, A\&A, 574, A129

Spite, M., Caffau, E., Bonifacio, P., Spite, F., Ludwig, H.-G., Plez, B., \& Christlieb, N., 2013, A\&A, 552, A107

Starkenburg, E. et al., 2013, A\&A, 549, A88

Starkenburg, E., Shetrone, M. D., McConnachie, A. W., \& Venn, K. A., 2014, MNRAS, 441, 1217

Suda, T., Aikawa M., Machida, M. N., Fujimoto, M. Y., \& Iben, Jr. I., 2004, ApJ, 611, 476

Umeda H. \& Nomoto K., 2003, Nature, 422, 871

Venn K. A. \& Lambert D. L., 2008, ApJ, 677, 572

Venn, K. A., Puzia, T. H., Divell, M., Côté, S., Lambert, D. L., \& Starkenburg, E., 2014, ApJ, 791, 98

Yanny, B. et al., 2009, AJ, 137, 4377

Yong, D. et al., 2013a, ApJ, 762, 26

-, 2013b, ApJ, 762, 27 